\title{
The Role of Exosomes and Exosomal MicroRNA in Cardiovascular Disease
}

\author{
Dongdong Zheng ${ }^{1}$, Ming Huo ${ }^{2}$, Bo Li', Weitie Wang', Hulin Piao', Yong Wang', \\ Zhicheng Zhu' ${ }^{1}$, Dan Li ${ }^{1}$, Tiance Wang ${ }^{1}$ and Kexiang Liu ${ }^{1 *}$ \\ ${ }^{1}$ Department of Cardiovascular Surgery, The Second Hospital of Jilin University, Changchun, China, ${ }^{2}$ Department of Day \\ Operating Room, The Second Hospital of Jilin University, Changchun, China
}

OPEN ACCESS

Edited by:

Jose Maria Carvajal-Gonzalez, University of Extremadura, Spain

Reviewed by: Lorenzo Sempere, Michigan State University,

United States

Hamed Mirzaei,

Kashan University of Medical

Sciences, Iran

Lucio Barile,

University of Zurich, Switzerland

*Correspondence: Kexiang Liu

kxliu64@hotmail.com

Specialty section:

This article was submitted to

Signaling,

a section of the journal

Frontiers in Cell and Developmental

Biology

Received: 11 October 2020

Accepted: 17 December 2020

Published: 12 January 2021

Citation:

Zheng $D$, Huo $M$, Li B, Wang W,

Piao H, Wang Y, Zhu Z, Li D, Wang T and Liu K (2021) The Role

of Exosomes and Exosomal

MicroRNA in Cardiovascular Disease.

Front. Cell Dev. Biol. 8:616161.

doi: 10.3389/fcell.2020.616161
Exosomes are small vesicles (30-150 nm in diameter) enclosed by a lipid membrane bilayer, secreted by most cells in the body. They carry various molecules, including proteins, lipids, mRNA, and other RNA species, such as long non-coding RNA, circular RNA, and microRNA (miRNA). miRNAs are the most numerous cargo molecules in the exosome. They are endogenous non-coding RNA molecules, approximately 19-22-ntlong, and important regulators of protein biosynthesis. Exosomes can be taken up by neighboring or distant cells, where they play a role in post-transcriptional regulation of gene expression by targeting mRNA. Exosomal miRNAs have diverse functions, such as participation in inflammatory reactions, cell migration, proliferation, apoptosis, autophagy, and epithelial-mesenchymal transition. There is increasing evidence that exosomal miRNAs play an important role in cardiovascular health. Exosomal miRNAs are widely involved in the occurrence and development of cardiovascular diseases, such as atherosclerosis, acute coronary syndrome, heart failure (HF), myocardial ischemia reperfusion injury, and pulmonary hypertension. In this review, we present a systematic overview of the research progress into the role of exosomal miRNAs in cardiovascular diseases, and present new ideas for the diagnosis and treatment of cardiovascular diseases.

Keywords: exosome, microRNA, cardiovascular disease, communication, post transcriptional regulation

\section{INTRODUCTION}

Exosomes were originally identified in differentiated reticulocytes by Pan et al. (1985). The authors demonstrated that transferrin and many membrane-associated proteins are secreted from the cell in a vesicle during reticulocyte maturation, and they defined this vesicle as an exosome. At first, exosomes were thought to be involved in waste excretion from the cell, and they remained largely

Abbreviations: AAA, abdominal aortic aneurysm; ACS, acute coronary syndrome; AGO, argonaute protein; AMI, acute myocardial infarction; BMSC, bone marrow mesenchymal stem cell; CF, cardiac fibroblast; CPC, cardiac progenitor cells; CDC, cardiophere-derived cells; DGCR8, DiGeorge Syndrome critical region 8 homolog; ESCRT, endosomal-sorting complex required for transport; GW182, glycine-tryptophan protein of 182 Da; HF, heart failure; hnRNP, heterogeneous nuclear ribonucleoprotein; H/R, hypoxia and reoxygenation; ILV, intraluminal vesicle; lncRNA, long non-coding RNA; MACE, major adverse cardiovascular event; MAC, macrophage; MHC, major histocompatibility complex; MIRI, myocardial ischemiareperfusion injury; miRNA, microRNA; MSC, mesenchymal stem cell; MVB, multivesicular body; PAH, pulmonary arterial hypertension; PASMC, pulmonary artery smooth muscle cells; pri-miRNA, primary precursor of miRNAs; RHD, rheumatic heart disease; RISC, RNA-induced silencing complex; ROC, receiver operating characteristic; RVD, rheumatic valvular disease; SNARE, soluble $N$-ethylmaleimide-sensitive factor attachment protein receptor; TAA, thoracic aortic aneurysm; TLR, toll-like receptor; UA, unstable angina pectoris; VC, vascular calcification; VSMC, vascular smooth muscle cell. 
unexplored in the following decade. This changed in 1996, when Raposo et al. (1996) showed that exosomes from human and mouse $\mathrm{B}$ lymphocytes induce the response of antigenspecific major histocompatibility complex (MHC) II-restricted T lymphocytes, which indicated that these vesicles play an antigenpresenting role. Since then, exosomes have received increasing attention from the researchers, and it has been suggested that they may be a means of information transmission between cells. With the continuing research, diverse functions of exosomes have been discovered, such as participation in the immune response, antigen presentation, tumor cell migration and proliferation, apoptosis, autophagy, and so on (Bobrie et al., 2011; Hannafon and Ding, 2013; Robbins et al., 2016; Spees et al., 2016; Harada et al., 2017; Papandreou and Tavernarakis, 2017; Lindenbergh and Stoorvogel, 2018; Zeng et al., 2018; Zhang S. et al., 2018). In recent years, a large number of studies have shown that miRNAs use exosomes as carriers to achieve cell-to-cell communication (Asgarpour et al., 2020). Specifically, exosome miRNAs are secreted by donor cells in the form of paracrine or remote secretion, and then taken up by donor cells. The exosome miRNAs enter the recipient cells, and form a RISC complex by targeting the mRNA $3^{\prime} \mathrm{UTR}$ and $\mathrm{AGO} 2$ protein in the recipient cell to degrade the mRNA of the target gene or inhibit the process of mRNA translation into protein. For example, Barile et al. (2014) showed that among exosome miRNAs secreted by cardiac progenitor cells (CPCs), the miRNAs with the highest content include miR-210, miR-132, and miR-146a-3p. Among them, after the exosome miR-210 is taken up by cardiomyocytes, it can down-regulate the expression of ephrin A3 and PTP1b to inhibit cardiomyocyte apoptosis. After exosome miR-132 is taken up by umbilical vein endothelial cells, the expression of RabGAP-P120 can be down-regulated by targeting to promote endothelial cell formation into tubes (Barile et al., 2014). Besides, as exosomes can protect miRNAs from harsh environments, exosomes miRNAs can stably exist in human plasma and body fluids. Therefore, the structural stability of exosome miRNAs makes it a non-invasive biomarker for disease prognosis and disease monitoring, such as cervical cancer and malignant glioma (Ghaemmaghami et al., 2020; Nahand et al., 2020). With further research, exosome miRNAs can be used as biomarkers for the diagnosis and prognosis of acute cardiovascular events. In this review, we present an overview of the current state of the knowledge on exosome biogenesis and function, with a specific focus on exosomal microRNA (miRNA) involvement in cardiovascular diseases, and the potential of exosomal miRNAs as novel diagnosis and treatment tools.

\section{OVERVIEW OF EXOSOME FORMATION}

\section{Exosome Biogenesis}

Exosomes originate from the endosome system (Figure 1). The endosome membrane invaginates and sprouts to form intraluminal vesicles (ILVs), which are early endosomes. ILVs further mature to form multivesicular bodies (MVBs), which are late endosomes (Hessvik and Llorente, 2018; Gurunathan et al., 2019). The endosomal-sorting complex required for transport
(ESCRT) plays an important role in MVB formation. ESCRT complexes include ESCRT-0, ESCRT-I, ESCRT-II, ESCRT-III, and Vps4 (Schmidt and Teis, 2012). In addition, MVBs also form via an ESCRT-independent pathway (Marsh and van Meer, 2008). Stuffers et al. (2009) observed that ILVs form MVBs even after ESCRT inhibition, indicating the existence of such an ESCRT-independent mechanism for MVB formation. Transmembrane proteins that are abundant in exosomes are involved in this process. van Niel et al. (2011) knocked out the expression of CD63 in HEK293 cells and observed a significant reduction in MVB production. This indicated that the transmembrane protein CD69 plays an important role in mediating the formation of MVBs via an ESCRT-independent mechanism. Further, Chairoungdua et al. (2010) demonstrated that CD9 and CD82 also play an important role in the ESCRTindependent mechanism. The authors showed that CD9 and CD82 promote exosome $\beta$-catenin release.

According to current research, the sorting mechanism depending on the ESCRT-dependent pathway may be involved in lysosomal degradation of proteins in ILVs, while the sorting mechanism depending on the ESCRT-independent pathway may be involved in ILV secretion. However, the process of MVB formation is likely to be much more complicated, and may depend on the cell type, cargo, and stimulation conditions. In addition, the transformation between two mechanisms mentioned above is still unclear.

\section{MVB Transport}

Multivesicular bodies can reach lysosomes, where their contents are then degraded or transported to the plasma membrane to release exosomes. MVB transport to the cell membrane and fusion with the cell membrane depends on microtubules, actin cytoskeleton, t-soluble $\mathrm{N}$-ethylmaleimide-sensitive factor attachment protein receptor (SNARE), v-SNARE, and Rab GTPases. Sinha et al. (2016) showed that the actin skeleton regulator cortactin promotes exosome secretion. As revealed by live-cell imaging, cortactin controls MVB transport and cell membrane docking. Cortactin, Rab27, and coronin $1 \mathrm{~b}$ cooperatively control the stability of the docking site between actin MVB and the cell membrane, and exosome secretion (Sinha et al., 2016).

The exact mechanism of MVB fusion with the plasma membrane is not clear. The known proteins involved in the fusion are SNAREs, Rabs, and Ras GTPase. SNAREs play an important role in promoting the fusion of MVBs and target membranes. The SNARE family includes $v$-SNARE and t-SNARE (Bonifacino and Glick, 2004). Fader et al. (2009) reported that v-SNARE vesicle-associated membrane protein 7 is necessary for exosome release from the human leukemia cell line K562. However, little is known about the mechanism of fusion between MVBs and lysosomes. Villarroya-Beltri et al. (2016) proposed that ISGylation controls the fate of MVBs. ISGylation is a ubiquitin-like protein modification process. After induction of ISGylation, the number of MVBs in cells dramatically decreases, and the secretion of exosomes also notably decreases. Indeed, ISGylation triggers the fusion of MVBs and lysosomes, leading to the aggregation and degradation of MVB proteins in lysosomes. 


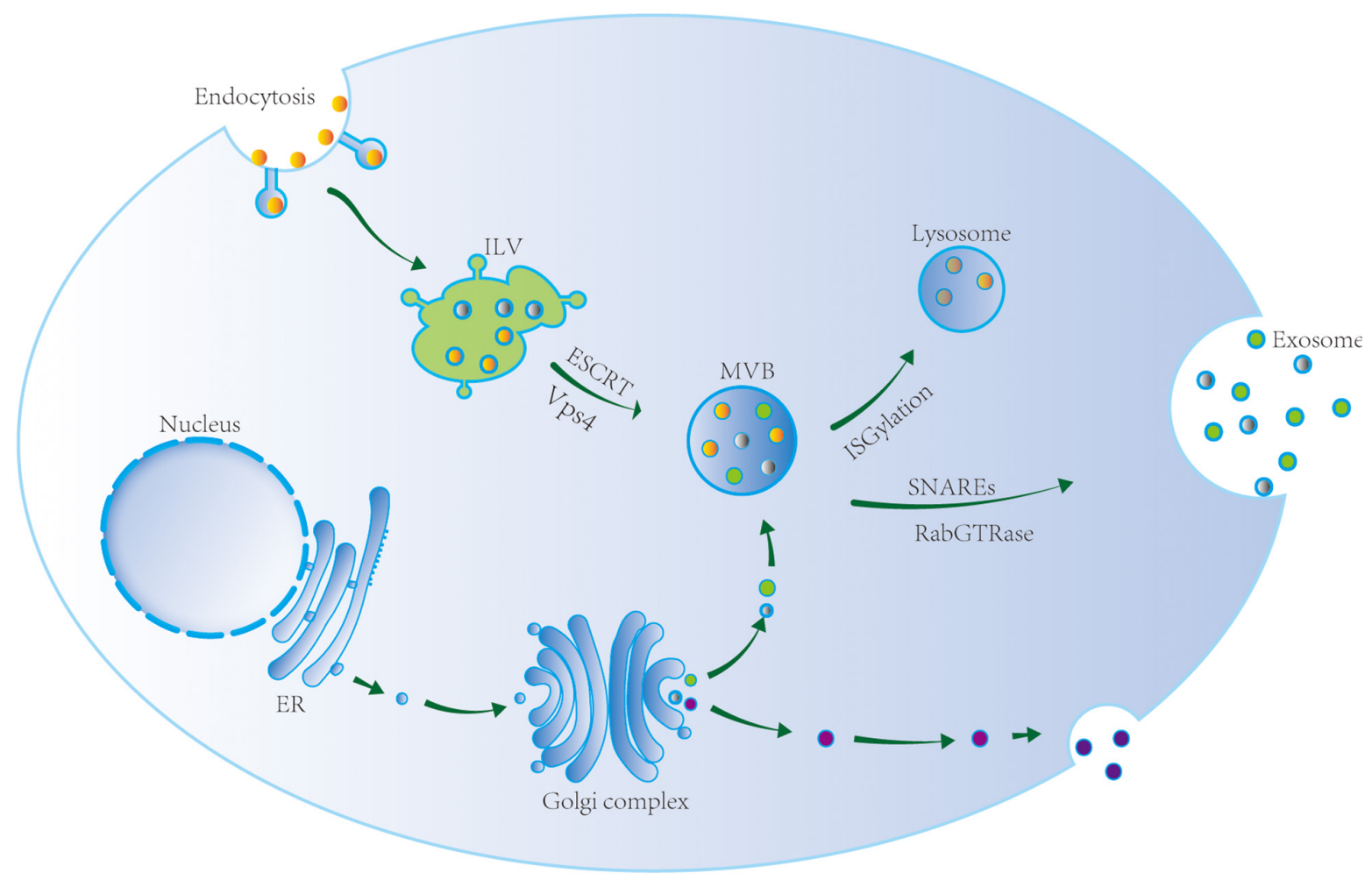

FIGURE 1 | Exosome biogenesis, sorting, and trafficking. Exosomes originate from the endosome system. The endosome membrane invaginates and sprouts to form intraluminal vesicle (ILV), the early endosome. ILV matures to form multivesicular body (MVB) via ESCRT and Vsp4, i.e., the late endosome. MVB reach Iysosome after ISGylation and its contents are degraded, or is transported to the cell membrane to release exosomes via SNAREs and RabGTPase.

Inhibition of lysosome function or autophagy can restore exosome secretion. These observations indicate that ISGylation is a new ubiquitination-like modification that controls the secretion of exosomes.

\section{EXOSOMAL MIRNAs}

\section{Biogenesis of miRNAs}

MicroRNAs are endogenous non-coding RNAs that are approximately 19-22-nt and play an important role in the regulation of protein biosynthesis. There are two main sources of miRNAs in eukaryotic cells. Most miRNAs are encoded by their own genes, transcribed by the RNA polymerase II, and processed to produce miRNAs. However, a small number of miRNA sequences are encoded within other RNA molecules. Following transcription of the corresponding RNA molecules, miRNAs can be produced via specific processing mechanisms, such as long non-coding RNA (lncRNA) (Figure 2).

In most cases, specific miRNA transcripts are generated by RNA polymerase II, which is a classic method of miRNA biosynthesis (Karube et al., 2005; Lin and Gregory, 2015; Wojciechowska et al., 2017; Lu and Rothenberg, 2018). Transcription produces pri-miRNA, the primary precursor of miRNA. Pri-miRNA is modified post-transcriptionally, with a cap structure added at the $5^{\prime}$-end and a polyA tail added at the $3^{\prime}$-end. The pri-miRNA is usually several thousand nucleotides long, and can form a stem-loop structure, with the mature miRNA sequence in the stem portion (the RNA fragments forming the stem are not entirely complementary). Pri-miRNA is processed in the nucleus by the RNase III family protein Drasha. In mammals, Drasha combines with the double-stranded RNAbinding protein DiGeorge Syndrome critical region 8 homolog (DGCR8) to form a Drasha-DGCR8 complex. The complex processes pri-miRNA into a miRNA precursor of approximately 70-nt, the pre-miRNA. Pre-miRNA has a hairpin structure (Lee et al., 2003; Gregory et al., 2004; Han et al., 2004; Merritt et al., 2008; Walz et al., 2015). It is transported to the cytoplasm by the export protein Exportin 5 (Yi et al., 2003). In the cytoplasm, Dicer, another member of the RNase III family, processes the pre-miRNA. Dicer binds to the double-stranded RNA-binding protein TRBP to form a complex that cleaves the pre-miRNA. The loop structure at the end of the double strand is cut off, producing a miRNA/miRNA* double-stranded molecule of approximately $20 \mathrm{bp}$ (Karube et al., 2005). After processing, if the two strands of short-chain RNA molecules are not completely complementary, they bind AGO 1 protein to form miRNARNA-induced silencing complex (miRISC). If the two strands of short-chain RNA molecules are complementary, they bind AGO2 protein to form another miRISC. Generally, miRNA* on one strand of the double-stranded RNA molecule is released and degraded, whereas miRNA on the other strand remains in the 


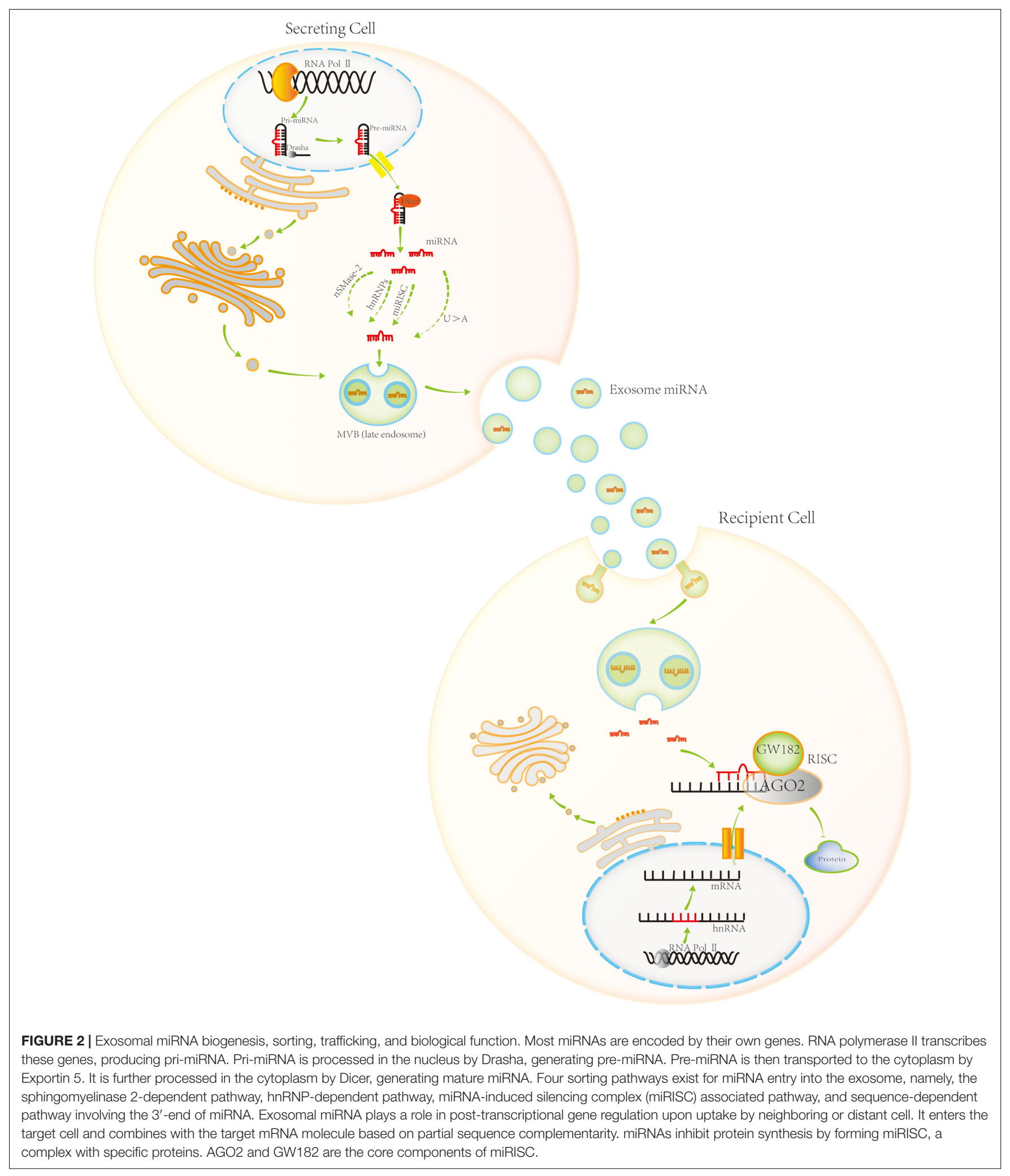

RISC complex. However, miRNA* is not always a byproduct of processing, and sometimes it is loaded into the RISC complex to play the role of miRNA (Jonas and Izaurralde, 2015; Michlewski and Cáceres, 2019).
Although most miRNAs are transcribed from specific miRNA genes, this is not the only way in which miRNAs are generated. Pre-miRNA sequences can also be found in introns of proteincoding genes or in other non-coding RNAs. Intron sequences 
of some protein-coding genes contain miRNA precursors, and transcribed RNA can form pri-miRNA containing a stemloop structure. Then, the Drosha-DGCR8 complex cuts primiRNA from the bottom of the stem-loop and releases premiRNA of approximately $70 \mathrm{nt}$. Some miRNA sequences are found in very short intron sequences. In this case, miRNA is produced from the intron sequences of protein-coding genes post-transcription. Specifically, miRNA is produced by an intron splicing mechanism, which does not require the participation of the Drosha-DGCR8 complex. The follow-up processing of pre-miRNAs from introns of protein-coding genes is the same as that for pre-miRNA from miRNA genes, and pre-miRNA is transported to the cytoplasm by Exportin 5. In the cytoplasm, it is further processed by the Dicer-TRBP complex to form miRNA/miRNA* double-strands of approximately $20 \mathrm{bp}$.

In addition, miRNAs can be generated from long noncoding (lnc) RNA. One of the functions of lncRNA may be to act as a small-RNA precursor (less than 200-nt-long). Some miRNAs are produced by lncRNA processing, usually after cutting of the lncRNA chain by the Drosha and Dicer enzymes (Achkar et al., 2016).

\section{Exosomal miRNA Sorting}

Exosomes contain a variety of molecules, including proteins, lipids, DNA, mRNA, miRNA, lncRNA, and circular RNA. Among them, exosomal miRNAs are the most numerous exosomal cargo molecules. They are involved in post-transcriptional regulation of gene expression, and have attracted much attention. Although the underlying mechanisms are not entirely clear, four possible pathways for miRNA sorting into exosomes have been identified (Figure 2). The first one is the sphingomyelinase 2-dependent pathway. Kosaka et al. (2013) demonstrated that sphingomyelinase 2 promotes the secretion of exosomal miRNA. The second pathway is the heterogeneous nuclear ribonucleoprotein (hnRNP)-dependent pathway. VillarroyaBeltri et al. (2013) showed that hnRNPA2B1 controls exosomal miRNAs entry to the exosomes. The third pathway is the miRISC-associated pathway. Therein, mature miRNA interacts with the assembly proteins to form RISC complexes. The miRISC complex is composed of miRNA, mRNA inhibited by the miRNA, AGO2, and glycine-tryptophan protein of $182 \mathrm{Da}$ (GW182). AGO2 preferentially binds to $U$ or $A$ at the end of miRNA, which plays an important role in mediating the formation of the miRNA:mRNA complex, and then degrading the mRNA molecule or blocking its translation. Frank et al. (2010), Guduric-Fuchs et al. (2012) observed that knocking out AGO2 in HEK293T cells results in a significant reduction in the exosomal miRNA release from the cells, such as exosomal miR-142-3p, miR-150, and miR-451. The final, fourth, pathway is the sequence-dependent pathway involving the $3^{\prime}$-end of miRNA. Lee et al. (2009), Koppers-Lalic et al. (2014) analyzed smallRNA profiles of human B cells and their secreted exosomes by RNA sequencing. Comprehensive bioinformatics and statistical analyses revealed that miRNAs are not randomly distributed in the B cells and their secreted exosomes. Specifically, miRNAs with an adenylated $3^{\prime}$-end are relatively enriched in the cells, while those with an uridylated $3^{\prime}$-end are relatively enriched in the exosomes. This indicates that the post-transcriptional modification of the $3^{\prime}$-end of miRNA plays an important role in guiding miRNAs into the exosome.

\section{Function of Exosomal miRNAs}

Once they are taken up by neighboring or distant cell, exosomal miRNAs play an important role in post-transcriptional regulation of gene expression. Exosomal miRNAs enter the target cell and combine with the mRNA of the target gene based on partial sequence complementarity. Seven nucleotides (nucleotides 2-8) at the $5^{\prime}$-end of miRNA comprise the seed region, which is key for mRNA recognition. The miRNA binding to a target mRNA regulates protein synthesis by forming miRISC.

Argonaute protein is the core component of RISC and directly binds miRNA. There are four AGO proteins in mammalian cells, AGO1-AGO4. The majority of miRNAs bind AGO1, while some bind AGO2. MiRISC formed by the combination of AGO1 and miRNA mainly inhibits protein translation, while miRISC formed by the combination of AGO2 and miRNA can shear target mRNA, resulting in gene expression silencing. GW182 protein is another important component of miRISC. They are recruited to miRNA targets through direct interactions with AGO2 proteins and promote target mRNA silencing.

The function of miRISC is to guide miRNA to bind to target mRNA. The complex regulates the translation of target mRNA in different ways. For example, Zeng et al. (2018) showed that in colorectal cancer, exosomal miR-25-3p is transferred from colorectal cancer cells to endothelial cells, where it regulates the expression of VEGFR2, ZO-1, occludin, and claudin5 by targeting KLF2 and KLF4, thus increasing vascular permeability and promoting angiogenesis. As another example, Hsu et al. (2017) demonstrated that exosomal miR-23 produced by lung cancer cells under hypoxia can promote angiogenesis and increase vascular permeability compact protein via ZO-1. In addition, Fabbri et al. (2012) observed that exosomal miR-21 and miR-29a secreted by tumor cells play a role in immune response, via another mechanism, i.e., by binding with toll-like receptors (TLRs) on immune cells. The binding triggers TLRmediated inflammatory reactions, ultimately promoting tumor cell proliferation and metastasis. Additional studies are needed to delineate this function of exosomal miRNA.

\section{CARDIOVASCULAR DISEASES AND EXOSOMAL MIRNAS}

\section{Atherosclerosis and Exosomal miRNAs}

Atherosclerosis is one of the most common vascular diseases, and the root cause of myocardial infarction and stroke. It is usually characterized by endothelial cell injury, aggregation of inflammatory cells and vascular smooth muscle cells (VSMCs), and deposition of extracellular lipids and fibrous tissue. Endothelial dysfunction is considered an early marker of atherosclerosis, which precedes the evidence of angiography or ultrasound of atherosclerotic plaques. Generally, endothelial cells are highly adaptable to injury stimuli, such as inflammatory stimuli and hyperlipidemia. Damaged endothelial cells are 
rapidly replaced by proliferating resident endothelial cells. However, as the injury stimulus persists, the endothelial cells undergo apoptosis and necrosis. Atherosclerosis occurs preferentially during laminar flow disorder, leading to endothelial cell injury, followed by chronic inflammation and VSMC proliferation. Under certain pathological conditions, the vascular wall cells are activated and highly proliferate, which resulted in the thickening and hardening of the arterial wall. Activated VSMCs play an important role in the progression of atherosclerosis. Therefore, preventing endothelial cell and VSMC dysfunction may reduce the risk of atherosclerosis-associated diseases, and provide a new treatment method.

Exosomal miRNAs play an important role in atherosclerosis and mediate intercellular communication (Libby et al., 2011; Hergenreider et al., 2012; Lovren et al., 2012; Gorenne et al., 2013; Hulsmans and Holvoet, 2013; Verweij et al., 2015; Blaser and Aikawa, 2019). Specifically, Hergenreider et al. (2012; Lovren et al., 2012) showed that exosomes secreted by human umbilical vein endothelial cells mediated by KLF2 stimulated by shear force are enriched in miR-143/145, which control the expression of target genes in co-cultured smooth muscle cells. Exosomes produced by endothelial cells overexpressing KLF2 reduce the formation of aortic sclerosis injury in $\operatorname{ApoE}(-/-)$ mouse. Further, according to an increasing number of studies, exosomal miRNAs of mesenchymal stem cells (MSCs) exert anti-atherosclerotic effects. For instance, Li J. et al. (2019) fed ApoE(-/-) mice highfat diet and used intravenous injection to administer exosomes from MSCs for 12 weeks in the mouse model. Following tail-vein injection, exosomes secreted by MSCs migrated to atherosclerotic plaques and resided near the macrophages. MSC exosomes reduced the atherosclerotic plaque area in these mice, greatly reduced the plaque infiltration by macrophages, and induced the polarization of macrophages to M2 type. In the same study, the enrichment of miR-let7 family in the MSC exosomes was increased. Endogenous miR-let7 levels increased in the aortic root of $\mathrm{ApoE}(-/-)$ mice, and the miR-let7 levels were further upregulated by MSC exosome treatment. In addition, knocking down miR-let7 expression in U937 cells inhibited cell migration and M2 polarization. Another study revealed that miR-let7 secreted by MSCs inhibits macrophage infiltration through the IGFZBP1/PTEN pathway and promotes the polarization of M2 macrophages through the HMGA2/NF- $\kappa$ B pathway.

The interaction between endothelial cells and macrophages mediated by exosomes plays an important role in the pathogenesis of atherosclerosis (Nguyen et al., 2018). Xing et al. (2020) showed that exosomal miR-342-5p from adiposederived MSCs exerts an anti-atherosclerotic effect on endothelial cells. Further, He et al. (2018) showed that the treatment of endothelial cells with oxidized low-density lipoprotein or overexpression of KLF2 results in enhanced exosomal miR-155 levels in endothelial cells. Exosomal miR-155 can polarize macrophages to M2 cells, which inhibit inflammatory reactions. In addition, Chang et al. (2019; Loyer et al., 2014) reported that the release of exosomal miR-92a upon endothelial cell injury increases during atherosclerosis. Exosomal miR-92a is taken up by macrophages in the local microenvironment, and activates macrophages by a targeted regulation of KLF4 to participate in the formation of atherosclerotic plaque. Zheng et al. (2017) showed that overexpression of KLF5 in smooth muscle cells promotes the expression of miR-155 and acts on adjacent endothelial cells, thus destroying the tight connection between endothelial cells and the integrity of endothelial barrier.

According to some recent studies, macrophages and VSMCs communicate with each other via exosomal miRNA during the onset of atherosclerosis. For example, Zhu et al. (2019) observed that stimulating macrophages with nicotine stimulates the release of exosomal miR-21-3p from macrophages. Exosomal miR-21-3p is taken up by neighboring VSMCs, promoting their proliferation and migration via targeted regulation of PTEN. Further, Liu et al. (2020) showed that oxidized low-density lipoprotein (ox-LDL) stimulates macrophages, which then secrete exosomal miR-106a$3 p$ to promote VSMC proliferation and inhibit VSMC apoptosis.

During the development of atherosclerosis, platelets can communicate with endothelial cells via exosomes as well. For example, Li et al. (2017) showed that the levels of miR-223, miR-339, and miR-21 in the exosomes released by platelets increase after thrombin activation of platelets. Exosomal miR-223 can induce the expression of ICAM-1 stimulated by TNF- $\alpha$ in endothelial cells, thus inhibiting inflammatory reaction.

In addition, dendritic cells and endothelial cells can also communicate with each other via exosomes. For example, Zhong et al. (2019) demonstrated that exosomal miR-146a secreted by dendritic cells is taken up by endothelial cells, where it regulates the inflammatory response by inhibiting IRAK-1.

\section{Acute Coronary Syndrome (ACS) and Exosomal miRNAs}

Acute coronary syndrome is a group of clinical syndromes whose pathological basis is the rupture or invasion of coronary atherosclerotic plaque, followed by complete or incomplete occlusive thrombosis, including ST-segment elevation myocardial infarction, acute non-ST segment elevation myocardial infarction, and unstable angina pectoris (UA). ACS is a common and serious coronary heart disease. ACS patients frequently experience paroxysmal chest pain, chest tightness, and other symptoms. ACS often leads to arrhythmia, HF, and even sudden death, hence seriously affecting the individual's quality of life. Receiving an appropriate treatment in a timely manner can greatly reduce the mortality and ACS complications, and improve the prognosis of patients.

According to recent studies, exosomal miRNAs play an important role in ACS. Generally speaking, it is anticipated that exosomal miRNAs will become an important tool in diagnosing and treating ACS (Ong et al., 2014; Sahoo and Losordo, 2014; Khan et al., 2015; Mathiasen et al., 2015; Saparov et al., 2017). For example, Bi et al. (2015) reported that exosomal miR-208a levels in the serum of ACS patients are significantly higher than those in healthy individuals. In that study, the average age of the ACS patient population analyzed $(n=500)$ was $62.35 \pm 9.70$ years, with 300 patients with low miR-208a levels and 200 patients with high miR-208a levels. The high-level miR-208a group was older, with higher Killip grade, and higher peak value of CK-MB than those in the low-level miR-208a group. After 1 year of follow-up, 
32 patients died, with 10 cases in the low-level miR-208a group (mortality rate of $3.3 \%$ ) and 22 patients in the high-level miR208a group (mortality rate of $11.0 \%$ ). According to the KaplanMeier survival analysis, the 1-year survival rate of patients with high miR-208a levels decreased significantly compared with low miR-208a level group. Further, Ling et al. (2020b) showed that the levels of miR-126 and miR-21 in UA and acute myocardial infarction (AMI) patients are significantly higher than those in healthy controls. According to the Gensini score, circulating exosomal miR-126 levels are positively correlated with coronary stenosis in patients with UA and AMI. Hence, miR-181a could be used as a new biomarker for diagnosing AMI.

Ling et al. (2020a) explored the exosomal miR-122-5p as a biomarker of UA and AMI, and asked whether its levels were positively correlated with the degree of coronary artery stenosis. Indeed, the exosomal miR-122-5p levels in the serum of patients with UA and AMI were significantly higher than those in the control group. Receiver operating characteristic (ROC) curve analysis revealed that the serum exosomal miR-122-5p levels could be used as a diagnostic marker for AMI and UA. In addition, according to the Gensini score, serum levels of miR122-5p were positively correlated with the degree of stenosis in UA patients. Therefore, increased levels of exosomal miR-122$5 \mathrm{p}$ in the serum can be used to predict the degree of coronary artery stenosis.

In addition, Xue et al. (2019) reported that miR-17-5p, miR126-5p, and miR-145-3p levels in the serum of patients with AMI were significantly elevated compared with health group. Based on ROC curve analysis, the miR-17-5p, miR-126-5p, and miR-145$3 p$ levels have a high diagnostic value for AMI. Similar, Faccini et al. (2017) reported that the levels of let-7c, miR-145c, and miR-155 in CAD patients were significantly lower than those in the control group. Accordingly, ROC curve analysis indicated that let-7c, miR-145c, or miR-155 levels were effective markers for CAD detection.

It has been also reported that, following AMI, damaged myocardial cells and mononuclear cells in the bone marrow can communicate with each other remotely via exosomal miRNA. For example, Saha et al. (2019) showed that injured myocardial cells release exosomal miR-1, exosomal miR-208, and exosome miR-499 after AMI, which then enter the bone marrow to downregulate the expression CXCR4 and increase the number of circulating monocytes.

Myocardial infarction is caused by myocardial ischemia, which is the main cause of morbidity and sudden death worldwide. In the process of myocardial infarction and subsequent HF, myocardial cells undergo remodeling, such as myocardial cell apoptosis, extracellular matrix protein increase, fibrosis, myocardial cell hypertrophy, and others. Therefore, the repair of myocardial cells and reversing myocardial remodeling are the key to treating HF. MSCs are considered to be a potential source of tissue-specific cells because of their pluripotency, low immunogenicity, availability, and expandability, and have been used in clinical trials to treat refractory diseases. Indeed, MSC transplantation can effectively reduce the left ventricular-end systolic volume and improve left ventricular ejection fraction in patients with severe ischemic HF. In-depth studies revealed that
MSCs repair damaged cardiomyocytes using exosomal miRNAs (Woodall and Gustafsson, 2018). For example, Zhu et al. (2018) showed that following hypoxia-induced MSCs, the release of exosomal miR-125b from MSCs increases. Exosomal miR-125b derived from MSCs effectively reduces the myocardial infarction area and promotes the repair of myocardial cells. In addition, research from the Luther group revealed that exosomal miR21a-5p secreted by MSCs plays a role in myocardial protection by down-regulating the expression of PDCD4, PTEN, Peli1, and FasL in cardiomyocytes (Wang et al., 2017; Luther et al., 2018). Similarly, Li Y. et al. (2019) demonstrated that exosomal miR301 derived from bone marrow MSCs (BMSCs) reduces the area of myocardial infarction and improves autophagy of myocardial cells. Further, Peng et al. (2020) showed that miR-25-3p secreted by MSCs reduces myocardial infarction area by targeting EZH2; Wen et al. (2020) showed that exosomal miR-144 derived from MSCs targets the regulation of the PTEN/AKT pathway, thus improving the apoptosis of cardiomyocytes under hypoxia. Geng et al. (2020) showed that miR-143, which is derived from serum exosomes, promotes myocardial angiogenesis through the IGFIR/NO pathway. Collectively, based on the above, exosomal miRNA is expected to become a new method for the diagnosis and treatment of ACS.

\section{Ischemia Reperfusion Injury and Exosomal miRNAs}

Myocardial ischemia reperfusion injury (MIRI) refers to the phenomenon in which the blood supply of the myocardial tissue is restored after a period of its interruption, but the injury from myocardial tissue is aggravated. The pathogenesis of MIRI has not been fully elucidated but it includes oxygen free-radical injury, intracellular calcium overload, and inflammatory injury (Ibáñez et al., 2015; Tibaut et al., 2017).

Recent studies have shown that exosomal miRNAs play an important role in MIRI. For example, Wang et al. (2019) showed that exosomal miR-126 reduces apoptosis in neonatal rat cardiomyocytes treated with $\mathrm{H}_{2} \mathrm{O}_{2}$ and $\mathrm{CoCl}_{2}$, and improves the cell survival rate. In addition, exosomal miR-126 significantly improves cardiac function. This indicates that exosomal miR126 can reduce MIRI by targeting and regulating ERRFI. Luo et al. (2019) showed that hypoxia and reoxygenation (H/R) significantly increase exosome secretion by cardiac fibroblasts (CFs) in a Transwell co-culture system, thus protecting H9C2 cells from H/R damage. Further analysis revealed that exosomal miR-423-3p secreted by CFs improves the viability of $\mathrm{H} 2 \mathrm{C} 9$ cells and reduces apoptosis by targeting RAP2C.

BMSCs are fibroblast-like pluripotent adult stem cells that exist in the bone marrow microenvironment. Injecting exosomes secreted by BMSCs into the infarcted area can significantly reduce the infarcted area and repair the function of myocardial cells after MIRI. According to recent studies, exosomes derived from BMSCs can promote the survival of cardiomyocytes and inhibit myocardial cell apoptosis after MIRI (Lu et al., 2015; Liu et al., 2017; Jiang et al., 2018). For example, Zhu et al. (2018) showed that exosomal miR-125b derived from BMSCs reduces MIRI by targeting SIRT7. Further, Sun et al. (2019) 
demonstrated that exosomes secreted by BMSCs induce the proliferation of $\mathrm{H} 9 \mathrm{C} 2$ cells and inhibit the apoptosis of $\mathrm{H} 9 \mathrm{C} 2$ cells in the H/R model, which indicates that these exosomes exert a protective effect against myocardial cell injury caused by H/R. In addition, exosomal miR-486-5p derived from BMSCs can repair myocardial injury caused by MIRI via PTEN/PI3K/Akt pathway. Zhao et al. (2019) showed that myocardial injection of BMSCs after MIRI effectively reduces the myocardial infarction area and alleviates the inflammatory reaction at the heart tissue and serum levels. The authors also found that miR-182, an exosome derived from BMSCs, promotes the polarization of M2 macrophages via TLR4, inhibits the inflammatory reaction, and plays a role in myocardial protection. Finally, it demonstrated that MSC-Exo reduces MIRI in mice via exosomal miR-182 that modifies the polarization status of macrophages. Therefore, based on the above, exosomal miRNAs derived from CFs and BMSCs are expected to become a new method for alleviating MIRI. In addition, recent studies have shown that exosomes derived from CDCs or cardiac-resident progenitor cells (CPCs) have a protective effect on cardiomyocytes. For example, Cambier et al. (2017) showed that exosomes Y RNA fragment (YF1) secreted by CDCs can reduce the area of myocardial infarction after MIRI. Ciullo et al. (2019) demonstrated that intramyocardial injection of exosomes derived from CPCs significantly reduced scars after myocardial infarction in rats and improved cardiac function. Barile et al. (2018) further compared the protective effects of exosomes derived from CPC and BMC on the myocardium. This study shows that exosomes derived from CPCs are more effective in reducing the size of scars and improving heart function after coronary artery occlusion in rats, compared with exosomes derived from BMCs (Barile et al., 2018). With further research, exosome miRNAs derived from CDCs or CPCs play an important role in myocardial protection. For example, Milano et al. (2020) showed that exosomes derived from CPCs miR146a-5p can effectively alleviate doxorubicin (Dox)/trastuzumab (Trz)-induced oxidative stress in cardiomyocytes. Ibrahim et al. (2014) showed that miR-146a derived from CDCs exosomes can reduce scar formation after myocardial infarction in rats, inhibit cardiomyocyte apoptosis, and improve heart function. Therefore, further in-depth study of exosomal miRNA derived from CDCs or CPCs provides a new direction for cardioprotective therapy.

\section{Heart Failure and Exosomal miRNAs}

Heart failure is caused by myocardial contraction dysfunction brought about by various factors. It leads to a decrease in cardiac blood output and blood stasis in the systemic or pulmonary circulation. $\mathrm{HF}$ is a major public health burden globally. Myocardial apoptosis, autophagy, inflammation, and myocardial cell remodeling are all involved in $\mathrm{HF}$. Ventricular remodeling refers to continuous myocardial injury, excessive mechanical load, and other factors that lead to the overexpression of inflammatory cytokines and overactivation of the neuroendocrine system, resulting in changes in the myocardial cell structure and function. The main pathological process of ventricular remodeling is myocardial cell thickening, ventricular volume enlargement, and cardiac cavity shape change, with the cardiac function changing from compensatory to decompensatory, which eventually leads to HF.

According to recent studies, exosomal miRNAs play an important role in myocardial remodeling (Danielson et al., 2018). Wang et al. (2018) reported altered levels of serum exosomal miR21, miR-425, and miR-744 in 31 patients with HF. The authors also observed down-regulation of miR-425 and miR-744 levels in CFs treated with angiotensin. The decrease in miR-425 and miR-744 levels was associated with the increase in collagen type 1 and $\alpha$-SMA expression, which led to the activation of CFs. Further analysis indicated that miR-425 and miR-744 inhibit the synthesis of collagen and cellulose induced by angiotensin, and also inhibit myocardial remodeling, by targeting TGF- $\beta$. In addition, the miR-425 and miR-744 levels are altered in the serum of patients with HF; therefore, miR-425 and miR-744 are expected to be therapeutic targets for reversing myocardial remolding and markers for diagnosing HF.

Wu et al. (2018) observed that serum exosomal miRNA can be used as a biomarker of HF with reduced ejection fraction. The authors showed that the exosomal miR-92b-5p levels are increased in the serum of patients with acute HF, which was negatively correlated with the left ventricular ejection fraction. Therefore, the serum levels of exosomal miR-92b-5p can be used as a biomarker for diagnosing HF with a reduced ejection fraction. In summary, exosomal miRNAs are expected to become a novel tool for diagnosing and treating HF.

\section{Pulmonary Arterial Hypertension (PAH) and Exosomal miRNAs}

Pulmonary arterial hypertension is a rare disease characterized by remodeling of the distal pulmonary circulation vessels. Vascular remodeling caused by pulmonary hypertension includes proliferation and apoptosis of the pulmonary vascular endothelial cells and extracellular matrix deposition, which leads to the loss of peripheral arterioles and occlusion of residual vascular beds. With the progression of $\mathrm{PAH}$, pulmonary vascular resistance increases, resulting in right $\mathrm{HF}$ and death. Currently, some drugs are available that show certain selectivity for pulmonary vascular dilatation. These drugs improve pulmonary vascular resistance to a certain extent and delay the deterioration of PAH patients' condition (Mathew, 2014; Rabinovitch et al., 2014). However, it is likely that curing PAH will require a drug that reverses pulmonary vascular remodeling caused by pulmonary hypertension.

It is not clear how pulmonary hypertension causes pulmonary vascular remodeling. Altered metabolism of endogenous pulmonary vasodilators, growth factors, and inflammatory pathways in PAH has been confirmed, but it is not clear whether these abnormalities also lead to changes in cell signaling.

Exosomal miRNAs play an important role in reversing pulmonary hypertension (Klinger et al., 2020). In cardiovascular diseases, the miR-143/145 cluster is the best studied miRNA cluster. Indeed, miR-145 and miR-143 are widely involved in cardiovascular diseases, such as atherosclerosis, coronary heart disease, aortic dissection, valvular disease, and pulmonary hypertension (Caruso et al., 2012; Liu et al., 2013, 2019; Guo et al., 2016; Girdauskas et al., 2018; Shi et al., 2018; Sun et al., 2018; Yue et al., 2018; Li T. et al., 2019; Vacante et al., 2019). 
Deng et al. (2015) reported that exosomal miR-143 regulates the interaction between VSMCs and endothelial cells in PAH. The authors observed that miR-143 levels are significantly increased in pulmonary vascular tissue in an animal model of pulmonary hypertension. Deletion of the miR-143 gene in mouse prevented the development of $\mathrm{PAH}$ induced by hypoxia. In addition, activation of the miR-143/145 gene cluster involves an upstream promoter region, which includes many binding elements; the cluster is activated by PAH-associated signaling pathways. MiR-143 regulates migration and apoptosis of pulmonary artery smooth muscle cells (PASMCs). Exosomal miR-143 derived from PASMCs promotes the migration and angiogenesis of endothelial cells. This highlights the intercellular communication between PASMCs and pulmonary artery endothelial cells (PAECs) via exosomal miR-143 during the progression of pulmonary hypertension, indicating that miR-143 is an important regulator of PAH. Consequently, a treatment strategy targeting miR-143 may constitute a highly beneficial treatment for this disease. In addition, Sindi et al. (2020) demonstrated that exosomal miR-181a-5p and miR-324$5 \mathrm{p}$ reverse pulmonary vascular remodeling caused by $\mathrm{PAH}$. Based on these observations, exosomal miRNA might be a new therapeutic target for reversing vascular remodeling caused by PAH.

\section{Aortic Aneurysm and Exosomal miRNA}

Aortic aneurysm refers to an aneurysm-like expansion of the aorta, usually an increase of the diameter by more than $50 \%$. The most serious complication of aortic aneurysm is rupture of the aorta, which is life-threatening. Smoking, advanced age, and male sex are strong risk factors for the development of aortic aneurysm. Indeed, among individuals over 55 years old, the incidence of abdominal aortic aneurysm (AAA) is $4-7 \%$ among males and 1-2\% among females (Golledge et al., 2006; Moll et al., 2011; Sidloff et al., 2014). At present, the diagnosis of AAA involves monitoring the diameter of the aorta. The current treatment methods are open surgery and endovascular repair. However, to date, no clinical trials of drugs that could limit the progression or rupture of AAA have been reported. Therefore, exploring the mechanism of AAA initiation and progression may be a new target to limit the AAA progression or rupture.

As in other cardiovascular disease, exosomal miRNAs are involved in AAA. For example, Han et al. (2020) showed that, compared with healthy individuals, exosomal miR-106a levels are elevated in AAA patients. Further analysis revealed that exosomal miR-106a promotes apoptosis of VSMCs. In addition, exosomal miR-106a increases the levels of matrix metalloproteinases (including matrix metalloproteinases 2 and 12) secreted by VSMCs via a targeted regulation of TIMP-2, which promotes the progression of AAA.

Maegdefessel et al. (2014) reported that miR-24 is the key regulator of vascular inflammation and AAA pathology in a mouse AAA disease model, and human aortic tissue and plasma. MiR-24 regulates the synthesis of cytokines in M1 macrophages by targeting CHI3L1, promotes the migration of VSMCs, and stimulates the expression of vascular endothelial adhesion molecules. The authors demonstrated that regulation of
miR-24 levels alters the progression of AAA in animal models, and that miR-24 represents a new plasma biomarker of human AAA disease progression. Collectively, exosomes could become a new target for reversing the expansion of aortic aneurysm.

\section{Vascular Calcification (VC) and Exosomal miRNA}

Vascular calcification is a pathological calcification of blood vessels, caused by abnormal metabolism of calcium and phosphate, osteogenic differentiation, inflammation, and other factors. It is closely related to the occurrence of major adverse cardiovascular events (MACEs). VC usually occurs under the intima of the blood vessel wall. A large number of studies published recently have demonstrated that VC is closely associated with the atherosclerotic plaque rupture. According to some clinical studies, MACE is more likely to occur during moderate or severe calcification during coronary vascularization than during non/mild calcification. Therefore, reversing the therapeutic target of VC could be a feasible method for reducing the occurrence of MACE. Statins are the most widely used representative drugs for $\mathrm{VC}$ treatment. However, although statins can effectively reduce the levels of low-density lipoprotein, they cannot alleviate the process of coronary artery calcification. Therefore, identification of a new target for reversing VC therapy is urgently needed.

Exosomal miRNAs play an important role in the formation of VC. For example, Gui et al. (2012) showed that elevated levels of miR-135, miR-762, miR-714, and miR-712 in VSMCs participate in VC by interfering with calcium efflux protein. Further, Xia et al. (2015) showed that the Runx2/miR-3960/miR2861 positive feedback loop is responsible for the transformation of VSMCs into osteoblasts and promotes the formation of VC. It has been also reported that exosomal miRNAs are involved in the formation of VC (Kapustin and Shanahan, 2016; Liberman and Marti, 2017; Zhang C. et al., 2018). For example, Pan et al. (2020) established a calcification model in the rat smooth muscle and MOVAS-1 cell line. The exosomal let-7e-5p levels decreased and exosomal miR-324-3p levels increased in the MOVAS-1 cell calcification model. Further analysis indicated that miR324-3p regulates MOVAS-1 calcification via IGF1R, PI3KCA, and MAP2K1. Therefore, exosomes may be developed into a new-generation targeted drugs to prevent $\mathrm{VC}$ and promote the stability of atherosclerotic plaques.

\section{Rheumatic Valvular Disease (RVD) and Exosomal miRNA}

Rheumatic heart disease (RHD) is a major problem in developing countries and the main cause of cardiovascular death among young people. It is an autoimmune disease caused by an abnormal antigen cross-reaction between group A streptococci and human connective tissue. RVD refers to the abnormal function of the valve and hemodynamic disturbances caused by repeated episodes of rheumatic carditis involving the heart valve and its accessory structures. Severe valve deformity results in high mortality. RVD most often involves the mitral valve, followed by the aortic valve, the latter often with the mitral valve damaged 


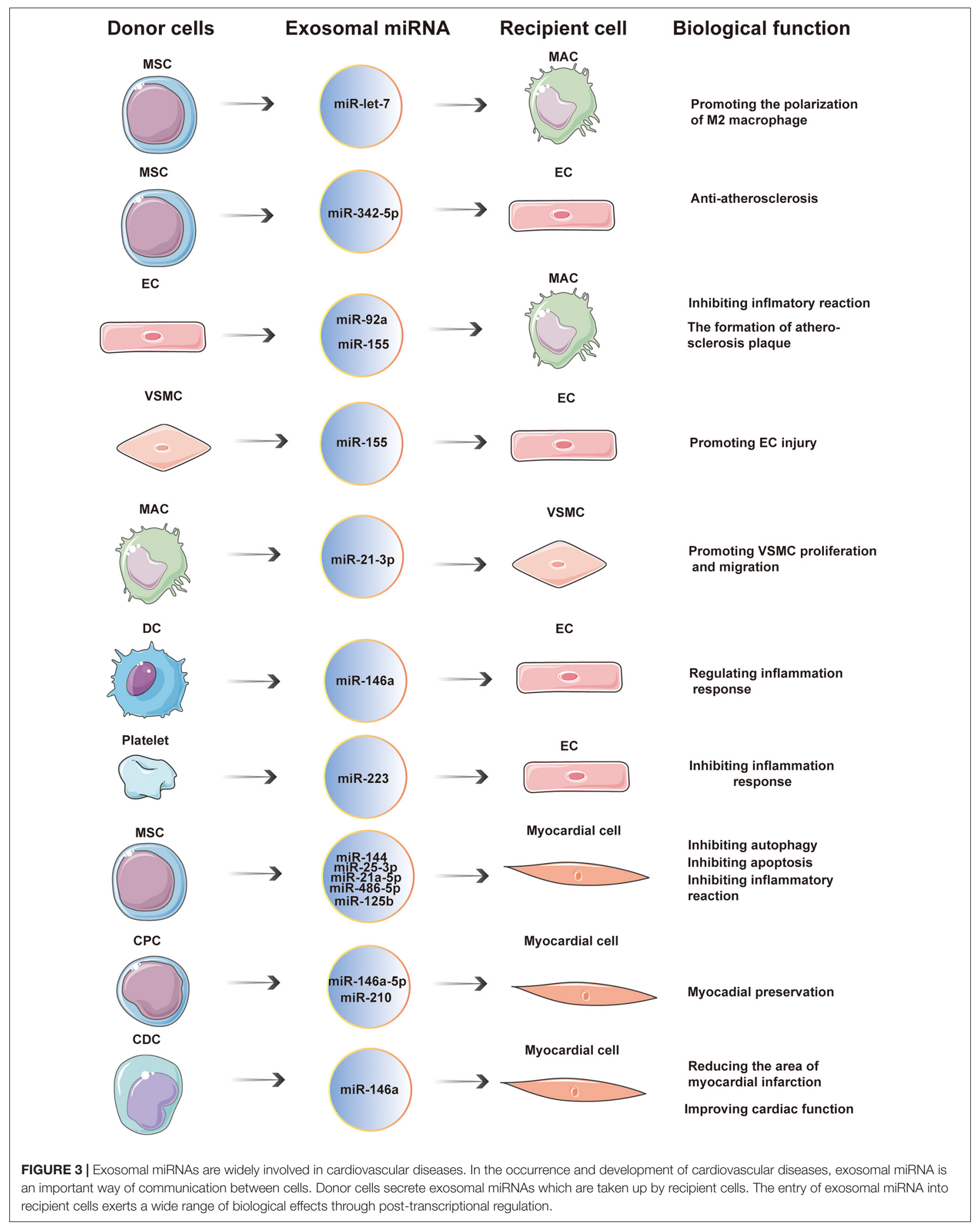


TABLE 1 | The role of exosomal miRNA in cardiovascular disease.

\begin{tabular}{|c|c|c|c|c|c|c|}
\hline miRNA & Donor cell & Recipient cell & Pathway & Disease & Function & References \\
\hline miR-155 & ECs & MACs & $\mathrm{KLF} 2 / \mathrm{miR}-155$ & AS & Inhibiting inflammatory reaction & He et al. (2018) \\
\hline miR-92a & ECs & MACs & Targeting KLF4 & AS & $\begin{array}{l}\text { The formation of atherosclerotic } \\
\text { plaque }\end{array}$ & $\begin{array}{l}\text { Loyer et al. (2014), Chang et al. } \\
\text { (2019) }\end{array}$ \\
\hline miR-106a-3p & MACs & VSMCs & N/A & AS & $\begin{array}{l}\text { Promoting VSMCs proliferation } \\
\text { and inhibiting VSMCs apoptosis }\end{array}$ & Liu et al. (2020) \\
\hline $\mathrm{miR}-146 \mathrm{a}$ & DCs & ECs & Targeting IRAK-1 & AS & $\begin{array}{l}\text { Regulating inflammation } \\
\text { response }\end{array}$ & Zhong et al. (2019) \\
\hline miR-301 & MSCs & Myocardial cells & $\mathrm{N} / \mathrm{A}$ & AMl & $\begin{array}{l}\text { Inhibiting autophagy of } \\
\text { myocardial cells }\end{array}$ & Li Y. et al. (2019) \\
\hline miR-25-3p & MSCs & Myocardial cells & Targeting E2Z2 & AMI & $\begin{array}{l}\text { Reducing the area of } \\
\text { myocardial infarction }\end{array}$ & Peng et al. (2020) \\
\hline miR-144 & MSCs & Myocardial cells & PTEN/AKT & AMI & $\begin{array}{l}\text { Improving the apoptosis of } \\
\text { cardiomyocytes under hypoxia }\end{array}$ & Wen et al. (2020) \\
\hline miR-126 & $\mathrm{N} / \mathrm{A}$ & Myocardial cells & Targeting ERRHI1 & AMI & $\begin{array}{l}\text { Improving myocardial cell } \\
\text { survival rate }\end{array}$ & Wang et al. (2019) \\
\hline miR-423-3p & $\begin{array}{l}\text { Cardiac } \\
\text { fibroblasts cells }\end{array}$ & Myocardial cells & Targeting RAP2C & MIRI & $\begin{array}{l}\text { Reducing myocardial cells } \\
\text { apoptosis }\end{array}$ & Luo et al. (2019) \\
\hline miR-486-5p & MSCs & Myocardial cells & PTEN/PI3K/Akt & $\mathrm{MIRI}$ & Repairing myocardial injury & Sun et al. (2019) \\
\hline let-7e-5p & $\mathrm{N} / \mathrm{A}$ & VSMCs & $\begin{array}{l}\text { IGF1R, PI3KCA and } \\
\text { MAP2K1 }\end{array}$ & VC & Regulating vascular calcification & Pan et al. (2020) \\
\hline miR-324-3p & $\mathrm{N} / \mathrm{A}$ & VSMCs & $\begin{array}{l}\text { IGF1R, PI3KCA and } \\
\text { MAP2K1 }\end{array}$ & VC & Regulating vascular calcification & Pan et al. (2020) \\
\hline$m i R-155-5 p$ & $N / A$ & N/A & S1PR1, SOCS1/STAT3 & RVD & $\begin{array}{l}\text { Regulating inflammation and } \\
\text { fibrosis }\end{array}$ & Chen et al. (2020) \\
\hline
\end{tabular}

TABLE 2 | The role of serum exosomal miRNAs in cardiovascular disease.

\begin{tabular}{lcccc}
\hline miRNA & Expression & Disease & Function & References \\
\hline miR-208a & $\uparrow$ & ACS & Diagnostic and prognostic & Bi et al. (2015) \\
miR-126 & $\uparrow$ & ACS & Diagnostic & Liagnostic \\
miR-21 & $\uparrow$ & ACS & Therapeutic & Ging et al. (2020b) \\
miR-143 & $\downarrow$ & AMl & Diagnostic and therapeutic & Wang et al. (2020) \\
miR-425 & $\downarrow$ & (2018) & Wang et al. (2018) \\
miR-744 & $\downarrow$ & HF & Diagnostic and therapeutic & Wu et al. (2018) \\
miR-92b-5p & $\uparrow$ & HF & Diagnostic & \\
\hline
\end{tabular}

$(\uparrow)$ and $(\downarrow)$ indicate increased and decreased expression of miRNA, respectively. 
at the same time, which is known as the combined valvular disease (Chopra and Gulwani, 2007; Marijon et al., 2012; Liu et al., 2015). According to recent studies, the S1PR1/STAT3 signaling pathway participates in RHD-induced cardiac valve injury by regulating Th17 cells (Wu et al., 2019). Further studies revealed that exosomal miRNA is involved in the regulation of this pathway during the development of RHD. Chen et al. (2020) observed that the serum levels of exosomal miR-155-5p are closely related to valve injury. Indeed, the exosomal miR-155$5 \mathrm{p}$ levels are increased in an RHD rat model. Further analysis revealed that exosomal miR-155-5p enhances the expression of S1PR1 and inhibits the activation of the SOCS1/STAT3 signaling pathway, thereby reducing the degree of valve inflammation and fibrosis, and reducing the levels of IL- 6 and IL-17 in the valve tissue and serum. These observations suggest that inhibition of miR-155-5p can reduce RHD-induced valve impairment through the S1PR1, SOCS1/STAT3, and IL-6/STAT3 signaling pathways. Although the role of exosomal miRNA in RVD has not been extensively studies, exosomal miRNA is nonetheless a promising and important tool for the treatment and diagnosis of RVD.

\section{CONCLUSION}

In conclusion, exosomal miRNAs play an important role in cardiovascular diseases. These molecules are widely involved in the occurrence and development of cardiovascular diseases, such as atherosclerosis, ACS, HF, myocardial infarction, and

\section{REFERENCES}

Achkar, N. P., Cambiagno, D. A., and Manavella, P. A. (2016). miRNA biogenesis: a dynamic pathway. Trends Plant Sci. 21, 1034-1044. doi: 10.1016/j.tplants.2016. 09.003

Asgarpour, K., Shojaei, Z., Amiri, F., Ai, J., Mahjoubin-Tehran, M., Ghasemi, F., et al. (2020). Exosomal microRNAs derived from mesenchymal stem cells: cell-to-cell messages. Cell Commun. Signal. 18:149. doi: 10.1186/s12964-02000650-6

Barile, L., Cervio, E., Lionetti, V., Milano, G., Ciullo, A., Biemmi, V., et al. (2018). Cardioprotection by cardiac progenitor cell-secreted exosomes: role of pregnancy-associated plasma protein-A. Cardiovasc. Res. 114, 992-1005. doi: 10.1093/cvr/cvy055

Barile, L., Lionetti, V., Cervio, E., Matteucci, M., Gherghiceanu, M., Popescu, L. M., et al. (2014). Extracellular vesicles from human cardiac progenitor cells inhibit cardiomyocyte apoptosis and improve cardiac function after myocardial infarction. Cardiovasc. Res. 103, 530-541. doi: 10.1093/cvr/cvu167

Bi, S., Wang, C., Jin, Y., Lv, Z., Xing, X., and Lu, Q. (2015). Correlation between serum exosome derived miR-208a and acute coronary syndrome. Int. J. Clin. Exp. Med. 8, 4275-4280.

Blaser, M. C., and Aikawa, E. (2019). Differential miRNA loading underpins dual harmful and protective roles for extracellular vesicles in atherogenesis. Circ. Res. 124, 467-469. doi: 10.1161/CIRCRESAHA.119.314596

Bobrie, A., Colombo, M., Raposo, G., and Théry, C. (2011). Exosome secretion: molecular mechanisms and roles in immune responses. Traffic. 12, 1659-1668. doi: $10.1111 / \mathrm{j} .1600-0854.2011 .01225 . \mathrm{x}$

Bonifacino, J. S., and Glick, B. S. (2004). The mechanisms of vesicle budding and fusion. Cell 116, 153-166. doi: 10.1016/s0092-8674(03)01079-1

Cambier, L., de Couto, G., Ibrahim, A., Echavez, A. K., Valle, J., Liu, W., et al. (2017). Y RNA fragment in extracellular vesicles confers cardioprotection via modulation of IL-10 expression and secretion. EMBO Mol. Med. 9, 337-352. doi: 10.15252/emmm.201606924

Caruso, P., Dempsie, Y., Stevens, H. C., McDonald, R. A., Long, L., Lu, R., et al. (2012). A role for miR-145 in pulmonary arterial hypertension: evidence from pulmonary hypertension. Exosomal miRNAs are an important means of intercellular communication. As such, they can impede atherosclerosis, reduce MIRI, improve myocardial function after $\mathrm{HF}$, and regulate the inflammatory response (Figure 3 and Table 1). In addition, some exosomal miRNAs are differently expressed in the serum of patients with atherosclerosis, HF, ACS, and other cardiovascular diseases, compared with healthy patients, and are closely related to the diagnosis and severity of these diseases (Table 2). Therefore, exosomal miRNAs are expected to be developed into a novel tool for diagnosing and treatment of cardiovascular diseases.

\section{AUTHOR CONTRIBUTIONS}

$\mathrm{DZ}$ and KL researched the article and wrote the manuscript. $\mathrm{MH}$, BL, WW, HP, YW, ZZ, DL, and TW reviewed and edited the manuscript before submission. All authors provided substantial contribution to the discussion of content.

\section{FUNDING}

This work was supported by the National Natural Science Foundation of China, Grant No. 81970399 and supported by Key Laboratory construction project of Jilin Province, Grant No. 20190901008JC.

mouse models and patient samples. Circ. Res. 111, 290-300. doi: 10.1161/ CIRCRESAHA.112.267591

Chairoungdua, A., Smith, D. L., Pochard, P., Hull, M., and Caplan, M. J. (2010). Exosome release of $\beta$-catenin: a novel mechanism that antagonizes Wnt signaling. J. Cell Biol. 190, 1079-1091. doi: 10.1083/jcb.201002049

Chang, Y. J., Li, Y. S., Wu, C. C., Wang, K. C., Huang, T. C., Chen, Z., et al. (2019). Extracellular MicroRNA-92a mediates endothelial cell-macrophage communication. Arterioscler. Thromb. Vasc. Biol. 39, 2492-2504. doi: 10.1161/ ATVBAHA.119.312707

Chen, A., Wen, J., Lu, C., Lin, B., Xian, S., Huang, F., et al. (2020). Inhibition of miR155-5p attenuates the valvular damage induced by rheumatic heart disease. Int. J. Mol. Med. 45, 429-440. doi: 10.3892/ijmm.2019.4420

Chopra, P., and Gulwani, H. (2007). Pathology and pathogenesis of rheumatic heart disease. Indian J. Pathol. Microbiol. 50, 685-697.

Ciullo, A., Biemmi, V., Milano, G., Bolis, S., Cervio, E., Fertig, E. T., et al. (2019). Exosomal expression of CXCR4 targets cardioprotective vesicles to myocardial infarction and improves outcome after systemic administration. Int. J. Mol. Sci. 20:468. doi: 10.3390/ijms20030468

Danielson, K. M., Shah, R., Yeri, A., Liu, X., Camacho Garcia, F., Silverman, M., et al. (2018). Plasma circulating extracellular RNAs in left ventricular remodeling post-myocardial infarction. EBioMedicine. 32, 172-181. doi: 10. 1016/j.ebiom.2018.05.013

Deng, L., Blanco, F. J., Stevens, H., Lu, R., Caudrillier, A., McBride, M., et al. (2015). MicroRNA-143 activation regulates smooth muscle and endothelial cell crosstalk in pulmonary arterial hypertension. Circ Res. 117, 870-883. doi: 10.1161/CIRCRESAHA.115.306806

Fabbri, M., Paone, A., Calore, F., Galli, R., Gaudio, E., Santhanam, R., et al. (2012). MicroRNAs bind to Toll-like receptors to induce prometastatic inflammatory response. Proc. Natl. Acad. Sci. U.S.A. 109, E2110-E2116. doi: 10.1073/pnas. 1209414109

Faccini, J., Ruidavets, J. B., Cordelier, P., Martins, F., Maoret, J. J., Bongard, V., et al. (2017). Circulating miR-155, miR-145 and let-7c as diagnostic biomarkers of the coronary artery disease. Sci. Rep. 7:42916. doi: 10.1038/srep4 2916 
Fader, C. M., Sánchez, D. G., Mestre, M. B., and Colombo, M. I. T. I.-V. A. M. P. (2009). /VAMP7 and VAMP3/cellubrevin: two v-SNARE proteins involved in specific steps of the autophagy/multivesicular body pathways. Biochim. Biophys. Acta 1793, 1901-1916. doi: 10.1016/j.bbamcr.2009.09.011

Frank, F., Sonenberg, N., and Nagar, B. (2010). Structural basis for $5^{\prime}$-nucleotide base-specific recognition of guide RNA by human AGO2. Nature 465, 818-822. doi: 10.1038/nature09039

Geng, T., Song, Z. Y., Xing, J. X., Wang, B. X., Dai, S. P., and Xu, Z. S. (2020). Exosome derived from coronary serum of patients with myocardial infarction promotes angiogenesis through the miRNA-143/IGF-IR pathway. Int. J. Nanomed. 15, 2647-2658. doi: 10.2147/IJN.S242908

Ghaemmaghami, A. B., Mahjoubin-Tehran, M., Movahedpour, A., Morshedi, K., Sheida, A., Taghavi, S. P., et al. (2020). Role of exosomes in malignant glioma: microRNAs and proteins in pathogenesis and diagnosis. Cell Commun. Signal. 18:120. doi: 10.1186/s12964-020-00623-9

Girdauskas, E., Petersen, J., Neumann, N., Ungelenk, M., Kurth, I., Reichenspurner, H., et al. (2018). MiR-145 expression and rare NOTCH1 variants in bicuspid aortic valve-associated aortopathy. PLoS One 13:e0200205. doi: 10.1371/journal. pone.0200205

Golledge, J., Muller, J., Daugherty, A., and Norman, P. (2006). Abdominal aortic aneurysm: pathogenesis and implications for management. Arterioscler. Thromb. Vasc. Biol. 26, 2605-2613. doi: 10.1161/01.ATV.0000245819.32762.cb

Gorenne, I., Kumar, S., Gray, K., Figg, N., Yu, H., Mercer, J., et al. (2013). Vascular smooth muscle cell sirtuin 1 protects against DNA damage and inhibits atherosclerosis. Circulation 127, 386-396. doi: 10.1161/CIRCULATIONAHA. 112.124404

Gregory, R. I., Yan, K. P., Amuthan, G., Chendrimada, T., Doratotaj, B., Cooch, N., et al. (2004). The Microprocessor complex mediates the genesis of microRNAs. Nature 432, 235-240. doi: 10.1038/nature03120

Guduric-Fuchs, J., O’Connor, A., Camp, B., O’Neill, C. L., Medina, R. J., and Simpson, D. A. (2012). Selective extracellular vesicle-mediated export of an overlapping set of microRNAs from multiple cell types. BMC Genomics 13:357. doi: 10.1186/1471-2164-13-357

Gui, T., Zhou, G., Sun, Y., Shimokado, A., Itoh, S., Oikawa, K., et al. (2012). MicroRNAs that target $\mathrm{Ca}(2+)$ transporters are involved in vascular smooth muscle cell calcification. Lab. Invest. 92, 1250-1259. doi: 10.1038/labinvest. 2012.85

Guo, X., Yu, L., Chen, M., Wu, T., Peng, X., Guo, R., et al. (2016). miR-145 mediated the role of aspirin in resisting VSMCs proliferation and anti-inflammation through CD40. J. Transl. Med. 14:211. doi: 10.1186/s12967-016-0961-2

Gurunathan, S., Kang, M. H., Jeyaraj, M., Qasim, M., and Kim, J. H. (2019). Review of the isolation, characterization, biological function, and multifarious therapeutic approaches of exosomes. Cells 8:307. doi: 10.3390/cells8040307

Han, J., Lee, Y., Yeom, K. H., Kim, Y. K., Jin, H., and Kim, V. N. (2004). The Drosha-DGCR8 complex in primary microRNA processing. Genes Dev. 18, 3016-3027. doi: 10.1101/gad.1262504

Han, Z. L., Wang, H. Q., Zhang, T. S., He, Y. X., and Zhou, H. (2020). Up-regulation of exosomal miR-106a may play a significant role in abdominal aortic aneurysm by inducing vascular smooth muscle cell apoptosis and targeting TIMP-2, an inhibitor of metallopeptidases that suppresses extracellular matrix degradation. Eur. Rev. Med. Pharmacol. Sci. 24, 8087-8095. doi: 10.26355/eurrev_202008_ 22493

Hannafon, B. N., and Ding, W. Q. (2013). Intercellular communication by exosome-derived microRNAs in cancer. Int. J. Mol. Sci. 14, 14240-14269. doi: 10.3390/ijms140714240

Harada, T., Yamamoto, H., Kishida, S., Kishida, M., Awada, C., Takao, T., et al. (2017). Wnt5b-associated exosomes promote cancer cell migration and proliferation. Cancer Sci. 108, 42-52. doi: 10.1111/cas.13109

He, S., Wu, C., Xiao, J., Li, D., Sun, Z., and Li, M. (2018). Endothelial extracellular vesicles modulate the macrophage phenotype: potential implications in atherosclerosis. Scand. J. Immunol. 87:e12648. doi: 10.1111/sji.12648

Hergenreider, E., Heydt, S., Tréguer, K., Boettger, T., Horrevoets, A. J., Zeiher, A. M., et al. (2012). Atheroprotective communication between endothelial cells and smooth muscle cells through miRNAs. Nat. Cell. Biol. 14, 249-256. doi: $10.1038 /$ ncb2441

Hessvik, N. P., and Llorente, A. (2018). Current knowledge on exosome biogenesis and release. Cell Mol. Life Sci. 75, 193-208. doi: 10.1007/s00018-017-2595-9

Hsu, Y. L., Hung, J. Y., Chang, W. A., Lin, Y. S., Pan, Y. C., Tsai, P. H., et al. (2017). Hypoxic lung cancer-secreted exosomal miR-23a increased angiogenesis and vascular permeability by targeting prolyl hydroxylase and tight junction protein ZO-1. Oncogene 36, 4929-4942. doi: 10.1038/onc.2017.105

Hulsmans, M., and Holvoet, P. (2013). MicroRNA-containing microvesicles regulating inflammation in association with atherosclerotic disease. Cardiovasc. Res. 100, 7-18. doi: 10.1093/cvr/cvt161

Ibáñez, B., Heusch, G., Ovize, M., and Van de Werf, F. (2015). Evolving therapies for myocardial ischemia/reperfusion injury. J. Am. Coll. Cardiol. 65, 1454-1471. doi: 10.1016/j.jacc.2015.02.032

Ibrahim, A. G., Cheng, K., and Marbán, E. (2014). Exosomes as critical agents of cardiac regeneration triggered by cell therapy. Stem Cell Rep. 2, 606-619. doi: 10.1016/j.stemcr.2014.04.006

Jiang, X., Lew, K. S., Chen, Q., Richards, A. M., and Wang, P. (2018). Human mesenchymal stem cell-derived exosomes reduce ischemia/reperfusion injury by the inhibitions of apoptosis and autophagy. Curr. Pharm. Des. 24, 5334-5341. doi: $10.2174 / 1381612825666190119130441$

Jonas, S., and Izaurralde, E. (2015). Towards a molecular understanding of microRNA-mediated gene silencing. Nat. Rev. Genet. 16, 421-433. doi: 10.1038/ $\operatorname{nrg} 3965$

Kapustin, A. N., and Shanahan, C. M. (2016). Emerging roles for vascular smooth muscle cell exosomes in calcification and coagulation. J. Physiol. 594, 29052914. doi: 10.1113/JP271340

Karube, Y., Tanaka, H., Osada, H., Tomida, S., Tatematsu, Y., Yanagisawa, K., et al. (2005). Reduced expression of Dicer associated with poor prognosis in lung cancer patients. Cancer Sci. 96, 111-115. doi: 10.1111/j.1349-7006.2005.00015.x

Khan, M., Nickoloff, E., Abramova, T., Johnson, J., Verma, S. K., Krishnamurthy, P., et al. (2015). Embryonic stem cell-derived exosomes promote endogenous repair mechanisms and enhance cardiac function following myocardial infarction. Circ. Res. 117, 52-64. doi: 10.1161/CIRCRESAHA.117.30 5990

Klinger, J. R., Pereira, M., Del Tatto, M., Brodsky, A. S., Wu, K. Q., Dooner, M. S., et al. (2020). Mesenchymal stem cell extracellular vesicles reverse sugen/hypoxia pulmonary hypertension in rats. Am. J. Respir. Cell Mol. Biol. 62, 577-587. doi: 10.1165/rcmb.2019-0154OC

Koppers-Lalic, D., Hackenberg, M., Bijnsdorp, I. V., van Eijndhoven, M. A. J., Sadek, P., Sie, D., et al. (2014). Nontemplated nucleotide additions distinguish the small RNA composition in cells from exosomes. Cell Rep. 8, 1649-1658. doi: 10.1016/j.celrep.2014.08.027

Kosaka, N., Iguchi, H., Hagiwara, K., Yoshioka, Y., Takeshita, F., and Ochiya, T. (2013). Neutral sphingomyelinase 2 (nSMase2)-dependent exosomal transfer of angiogenic microRNAs regulate cancer cell metastasis. J. Biol. Chem. 288, 10849-10859. doi: 10.1074/jbc.M112.446831

Lee, Y., Ahn, C., Han, J., Choi, H., Kim, J., Yim, J., et al. (2003). The nuclear RNase III Drosha initiates microRNA processing. Nature 425, 415-419. doi: 10.1038/nature01957

Lee, Y. S., Pressman, S., Andress, A. P., Kim, K., White, J. L., Cassidy, J. J., et al. (2009). Silencing by small RNAs is linked to endosomal trafficking. Nat. Cell. Biol. 11, 1150-1156. doi: 10.1038/ncb1930

Li, J., Tan, M., Xiang, Q., Zhou, Z., and Yan, H. (2017). Thrombin-activated platelet-derived exosomes regulate endothelial cell expression of ICAM-1 via microRNA-223 during the thrombosis-inflammation response. Thromb. Res. 154, 96-105. doi: 10.1016/j.thromres.2017.04.016

Li, J., Xue, H., Li, T., Chu, X., Xin, D., Xiong, Y., et al. (2019). Exosomes derived from mesenchymal stem cells attenuate the progression of atherosclerosis in ApoE-/- mice via miR-let7 mediated infiltration and polarization of M2 macrophage. Biochem. Biophys. Res. Commun. 510, 565-572. doi: 10.1016/j. bbrc.2019.02.005

Li, T., Liu, C., Liu, L., Xia, H., Xiao, Y., Wang, X., et al. (2019). Regulatory mechanism of MicroRNA-145 in the pathogenesis of acute aortic dissection. Yonsei Med. J. 60, 352-359. doi: 10.3349/ymj.2019.60.4.352

Li, Y., Yang, R., Guo, B., Zhang, H., Zhang, H., Liu, S., et al. (2019). Exosomal miR-301 derived from mesenchymal stem cells protects myocardial infarction by inhibiting myocardial autophagy. Biochem. Biophys. Res. Commun. 514, 323-328. doi: 10.1016/j.bbrc.2019.04.138

Libby, P., Ridker, P. M., and Hansson, G. K. (2011). Progress and challenges in translating the biology of atherosclerosis. Nature 473, 317-325. doi: 10.1038/ nature 10146

Liberman, M., and Marti, L. C. (2017). Vascular calcification regulation by exosomes in the vascular wall. Adv. Exp. Med. Biol. 998, 151-160. doi: 10.1007/ 978-981-10-4397-0_10 
Lin, S., and Gregory, R. I. (2015). MicroRNA biogenesis pathways in cancer. Nat. Rev. Cancer 15, 321-333. doi: 10.1038/nrc3932

Lindenbergh, M. F. S., and Stoorvogel, W. (2018). Antigen presentation by extracellular vesicles from professional antigen-presenting cells. Annu. Rev. Immunol. 36, 435-459. doi: 10.1146/annurev-immunol-041015-055700

Ling, H., Guo, Z., Du, S., Liao, Y., Li, Y., Ding, C., et al. (2020a). Serum exosomal miR-122-5p is a new biomarker for both acute coronary syndrome and underlying coronary artery stenosis. Biomarkers 25, 539-547. doi: 10.1080/ 1354750X.2020.1803963

Ling, H., Guo, Z., Shi, Y., Zhang, L., and Song, C. (2020b). Serum exosomal MicroRNA-21, MicroRNA-126, and PTEN are novel biomarkers for diagnosis of acute coronary syndrome. Front. Physiol. 11:654. doi: 10.3389/fphys.2020. 00654

Liu, L., Jin, X., Hu, C. F., Li, R., Zhou, Z., and Shen, C. X. (2017). Exosomes derived from mesenchymal stem cells rescue myocardial ischaemia/reperfusion injury by inducing cardiomyocyte autophagy via AMPK and Akt pathways. Cell Physiol. Biochem. 43, 52-68. doi: 10.1159/000480317

Liu, M., Lu, L., Sun, R., Zheng, Y., and Zhang, P. (2015). Rheumatic heart disease: causes, symptoms, and treatments. Cell Biochem. Biophys. 72, 861-863. doi: 10.1007/s12013-015-0552-5

Liu, X., Cheng, Y., Yang, J., Qin, S., Chen, X., Tang, X., et al. (2013). Flank sequences of miR-145/143 and their aberrant expression in vascular disease: mechanism and therapeutic application. J. Am. Heart Assoc. 2:e000407. doi: 10.1161/JAHA. 113.000407

Liu, Y., Zhang, W. L., Gu, J. J., Sun, Y. Q., Cui, H. Z., Bu, J. Q., et al. (2020). Exosome-mediated miR-106a-3p derived from ox-LDL exposed macrophages accelerated cell proliferation and repressed cell apoptosis of human vascular smooth muscle cells. Eur. Rev. Med. Pharmacol. Sci. 24, 7039-7050. doi: 10. 26355/eurrev_202006_21697

Liu, Z., Tao, B., Fan, S., Pu, Y., Xia, H., and Xu, L. (2019). MicroRNA-145 protects against myocardial ischemia reperfusion injury via CaMKII-mediated antiapoptotic and anti-inflammatory pathways. Oxid. Med. Cell. Longev. 2019:8948657. doi: 10.1155/2019/8948657

Lovren, F., Pan, Y., Quan, A., Singh, K. K., Shukla, P. C., Gupta, N., et al. (2012). MicroRNA-145 targeted therapy reduces atherosclerosis. Circulation 126(11 Suppl. 1), S81-S90. doi: 10.1161/CIRCULATIONAHA.111.084186

Loyer, X., Potteaux, S., Vion, A. C., Guérin, C. L., Boulkroun, S., Rautou, P. E., et al. (2014). Inhibition of microRNA-92a prevents endothelial dysfunction and atherosclerosis in mice. Circ. Res. 114, 434-443. doi: 10.1161/CIRCRESAHA. 114.302213

Lu, T. X., and Rothenberg, M. E. (2018). MicroRNA. J. Allergy Clin. Immunol. 141, 1202-1207. doi: 10.1016/j.jaci.2017.08.034

Lu, W., Si, Y. I., Ding, J., Chen, X., Zhang, X., Dong, Z., et al. (2015). Mesenchymal stem cells attenuate acute ischemia-reperfusion injury in a rat model. Exp. Ther. Med. 10, 2131-2137. doi: 10.3892/etm.2015.2806

Luo, H., Li, X., Li, T., Zhao, L., He, J., Zha, L., et al. (2019). microRNA-423$3 p$ exosomes derived from cardiac fibroblasts mediates the cardioprotective effects of ischaemic post-conditioning. Cardiovasc. Res. 115, 1189-1204. doi: $10.1093 / \mathrm{cvr} / \mathrm{cvy} 231$

Luther, K. M., Haar, L., McGuinness, M., Wang, Y., Lynch Iv, T. L., Phan, A., et al. (2018). Exosomal miR-21a-5p mediates cardioprotection by mesenchymal stem cells. J. Mol. Cell. Cardiol. 119, 125-137. doi: 10.1016/j.yjmcc.2018. 04.012

Maegdefessel, L., Spin, J. M., Raaz, U., Eken, S. M., Toh, R., Azuma, J., et al. (2014). miR-24 limits aortic vascular inflammation and murine abdominal aneurysm development. Nat. Commun. 5:5214. doi: 10.1038/ncomms6214

Marijon, E., Mirabel, M., Celermajer, D. S., and Jouven, X. (2012). Rheumatic heart disease. Lancet 379, 953-964. doi: 10.1016/S0140-6736(11)61171-9

Marsh, M., and van Meer, G. (2008). Cell biology. No ESCRTs for exosomes. Science 319, 1191-1192. doi: 10.1126/science. 1155750

Mathew, R. (2014). Pathogenesis of pulmonary hypertension: a case for caveolin-1 and cell membrane integrity. Am. J. Physiol. Heart Circ. Physiol. 306, H15-H25. doi: 10.1152/ajpheart.00266.2013

Mathiasen, A. B., Qayyum, A. A., Jørgensen, E., Helqvist, S., Fischer-Nielsen, A., Kofoed, K. F., et al. (2015). Bone marrow-derived mesenchymal stromal cell treatment in patients with severe ischaemic heart failure: a randomized placebocontrolled trial (MSC-HF trial). Eur. Heart J. 36, 1744-1753. doi: 10.1093/ eurheartj/ehv136
Merritt, W. M., Lin, Y. G., Han, L. Y., Kamat, A. A., Spannuth, W. A., Schmandt, R., et al. (2008). Dicer, Drosha, and outcomes in patients with ovarian cancer. N. Engl. J. Med. 359, 2641-2650. doi: 10.1056/NEJMoa0803785

Michlewski, G., and Cáceres, J. F. (2019). Post-transcriptional control of miRNA biogenesis. RNA 25, 1-16. doi: 10.1261/rna.068692.118

Milano, G., Biemmi, V., Lazzarini, E., Balbi, C., Ciullo, A., Bolis, S., et al. (2020). Intravenous administration of cardiac progenitor cell-derived exosomes protects against doxorubicin/trastuzumab-induced cardiac toxicity. Cardiovasc. Res. 116, 383-392. doi: $10.1093 / \mathrm{cvr} / \mathrm{cvz} 108$

Moll, F. L., Powell, J. T., Fraedrich, G., Verzini, F., Haulon, S., Waltham, M., et al. (2011). Management of abdominal aortic aneurysms clinical practice guidelines of the European society for vascular surgery. Eur. J. Vasc. Endovasc. Surg. 41(Suppl. 1), S1-S58. doi: 10.1016/j.ejvs.2010.09.011

Nahand, J. S., Vandchali, N. R., Darabi, H., Doroudian, M., Banafshe, H. R., Moghoofei, M., et al. (2020). Exosomal microRNAs: novel players in cervical cancer. Epigenomics 12, 1651-1660. doi: 10.2217/epi-2020-0026

Nguyen, M. A., Karunakaran, D., Geoffrion, M., Cheng, H. S., Tandoc, K., Perisic Matic, L., et al. (2018). Extracellular vesicles secreted by atherogenic macrophages transfer MicroRNA to inhibit cell migration. Arterioscler. Thromb. Vasc. Biol. 38, 49-63. doi: 10.1161/ATVBAHA.117.309795

Ong, S. G., Lee, W. H., Huang, M., Dey, D., Kodo, K., Sanchez-Freire, V., et al. (2014). Cross talk of combined gene and cell therapy in ischemic heart disease: role of exosomal microRNA transfer. Circulation 130(11 Suppl. 1), S60-S69. doi: 10.1161/CIRCULATIONAHA.113.007917

Pan, B. T., Teng, K., Wu, C., Adam, M., and Johnstone, R. M. (1985). Electron microscopic evidence for externalization of the transferrin receptor in vesicular form in sheep reticulocytes. J. Cell Biol. 101, 942-948. doi: 10.1083/jcb.101.3. 942

Pan, W., Liang, J., Tang, H., Fang, X., Wang, F., Ding, Y., et al. (2020). Differentially expressed microRNA profiles in exosomes from vascular smooth muscle cells associated with coronary artery calcification. Int. J. Biochem. Cell. Biol. 118:105645. doi: 10.1016/j.biocel.2019.105645

Papandreou, M. E., and Tavernarakis, N. (2017). Autophagy and the endo/ exosomal pathways in health and disease. Biotechnol. J. 12:175. doi: 10.1002/ biot.201600175

Peng, Y., Zhao, J. L., Peng, Z. Y., Xu, W. F., and Yu, G. L. (2020). Exosomal miR-25$3 p$ from mesenchymal stem cells alleviates myocardial infarction by targeting pro-apoptotic proteins and EZH2. Cell Death Dis. 11:317. doi: 10.1038/s41419020-2545-6

Rabinovitch, M., Guignabert, C., Humbert, M., and Nicolls, M. R. (2014). Inflammation and immunity in the pathogenesis of pulmonary arterial hypertension. Circ. Res. 115, 165-175. doi: 10.1161/CIRCRESAHA.113.301141

Raposo, G., Nijman, H. W., Stoorvogel, W., Liejendekker, R., Harding, C. V., Melief, C. J., et al. (1996). B lymphocytes secrete antigen-presenting vesicles. J. Exp. Med. 183, 1161-1172. doi: 10.1084/jem.183.3.1161

Robbins, P. D., Dorronsoro, A., and Booker, C. N. (2016). Regulation of chronic inflammatory and immune processes by extracellular vesicles. J. Clin. Invest. 126, 1173-1180. doi: 10.1172/JCI81131

Saha, P., Sharma, S., Korutla, L., Datla, S. R., Shoja-Taheri, F., Mishra, R., et al. (2019). Circulating exosomes derived from transplanted progenitor cells aid the functional recovery of ischemic myocardium. Sci. Transl. Med. 11:eaau1168. doi: 10.1126/scitranslmed.aau1168

Sahoo, S., and Losordo, D. W. (2014). Exosomes and cardiac repair after myocardial infarction. Circ. Res. 114, 333-344. doi: 10.1161/CIRCRESAHA.114. 300639

Saparov, A., Ogay, V., Nurgozhin, T., Chen, W. C. W., Mansurov, N., Issabekova, A., et al. (2017). Role of the immune system in cardiac tissue damage and repair following myocardial infarction. Inflamm. Res. 66, 739-751. doi: 10.1007/ s00011-017-1060-4

Schmidt, O., and Teis, D. (2012). The ESCRT machinery. Curr. Biol. 22, R116R120. doi: 10.1016/j.cub.2012.01.028

Shi, L., Tian, C., Sun, L., Cao, F., and Meng, Z. (2018). The IncRNA TUG1/miR145-5p/FGF10 regulates proliferation and migration in VSMCs of hypertension. Biochem. Biophys. Res. Commun. 501, 688-695. doi: 10.1016/j.bbrc.2018.05.049

Sidloff, D., Stather, P., Dattani, N., Bown, M., Thompson, J., Sayers, R., et al. (2014). Aneurysm global epidemiology study: public health measures can further reduce abdominal aortic aneurysm mortality. Circulation 129, 747-753. doi: 10.1161/CIRCULATIONAHA.113.005457 
Sindi, H. A., Russomanno, G., Satta, S., Abdul-Salam, V. B., Jo, K. B., QaziChaudhry, B., et al. (2020). Therapeutic potential of KLF2-induced exosomal microRNAs in pulmonary hypertension. Nat. Commun. 11:1185. doi: 10.1038/ s41467-020-14966-x

Sinha, S., Hoshino, D., Hong, N. H., Kirkbride, K. C., Grega-Larson, N. E., Seiki, M., et al. (2016). Cortactin promotes exosome secretion by controlling branched actin dynamics. J. Cell. Biol. 214, 197-213. doi: 10.1083/jcb.201601025

Spees, J. L., Lee, R. H., and Gregory, C. A. (2016). Mechanisms of mesenchymal stem/stromal cell function. Stem Cell Res. Ther. 7:125. doi: 10.1186/s13287-0160363-7

Stuffers, S., Sem Wegner, C., Stenmark, H., and Brech, A. (2009). Multivesicular endosome biogenesis in the absence of ESCRTs. Traffic 10, 925-937. doi: 10. 1111/j.1600-0854.2009.00920.x

Sun, N., Meng, F., Xue, N., Pang, G., Wang, Q., and Ma, H. (2018). Inducible miR-145 expression by HIF-1a protects cardiomyocytes against apoptosis via regulating SGK1 in simulated myocardial infarction hypoxic microenvironment. Cardiol. J. 25, 268-278. doi: 10.5603/CJ.a2017.0105

Sun, X. H., Wang, X., Zhang, Y., and Hui, J. (2019). Exosomes of bone-marrow stromal cells inhibit cardiomyocyte apoptosis under ischemic and hypoxic conditions via miR-486-5p targeting the PTEN/PI3K/AKT signaling pathway. Thromb. Res. 177, 23-32. doi: 10.1016/j.thromres.2019.02.002

Tibaut, M., Mekis, D., and Petrovic, D. (2017). Pathophysiology of myocardial infarction and acute management strategies. Cardiovasc. Hematol. Agents Med. Chem. 14, 150-159. doi: 10.2174/1871525714666161216100553

Vacante, F., Denby, L., Sluimer, J. C., and Baker, A. H. (2019). The function of miR143, miR-145 and the MiR-143 host gene in cardiovascular development and disease. Vascul. Pharmacol. 112, 24-30. doi: 10.1016/j.vph.2018.11.006

van Niel, G., Charrin, S., Simoes, S., Romao, M., Rochin, L., Saftig, P., et al. (2011). The tetraspanin CD63 regulates ESCRT-independent and -dependent endosomal sorting during melanogenesis. Dev. Cell. 21, 708-721. doi: 10.1016/ j.devcel.2011.08.019

Verweij, S. L., van der Valk, F. M., and Stroes, E. S. (2015). Novel directions in inflammation as a therapeutic target in atherosclerosis. Curr. Opin. Lipidol. 26, 580-585. doi: 10.1097/MOL.0000000000000233

Villarroya-Beltri, C., Baixauli, F., Mittelbrunn, M., Fernández-Delgado, I., Torralba, D., Moreno-Gonzalo, O., et al. (2016). ISGylation controls exosome secretion by promoting lysosomal degradation of MVB proteins. Nat. Commun. 7:13588. doi: $10.1038 /$ ncomms 13588

Villarroya-Beltri, C., Gutiérrez-Vázquez, C., Sánchez-Cabo, F., Pérez-Hernández, D., Vázquez, J., Martin-Cofreces, N., et al. (2013). Sumoylated hnRNPA2B1 controls the sorting of miRNAs into exosomes through binding to specific motifs. Nat. Commun. 4:2980. doi: 10.1038/ncomms3980

Walz, A. L., Ooms, A., Gadd, S., Gerhard, D. S., Smith, M. A., Guidry Auvil, J. M., et al. (2015). Recurrent DGCR8, DROSHA, and SIX homeodomain mutations in favorable histology Wilms tumors. Cancer Cell. 27, 286-297. doi: 10.1016/j. ccell.2015.01.003

Wang, K., Jiang, Z., Webster, K. A., Chen, J., Hu, H., Zhou, Y., et al. (2017). Enhanced cardioprotection by human endometrium mesenchymal stem cells driven by exosomal MicroRNA-21. Stem Cells Transl. Med. 6, 209-222. doi: 10.5966/sctm.2015-0386

Wang, L., Liu, J., Xu, B., Liu, Y. L., and Liu, Z. (2018). Reduced exosome miR425 and miR-744 in the plasma represents the progression of fibrosis and heart failure. Kaohsiung J. Med. Sci. 34, 626-633. doi: 10.1016/j.kjms.2018.05.008

Wang, W., Zheng, Y., Wang, M., Yan, M., Jiang, J., and Li, Z. (2019). Exosomes derived miR-126 attenuates oxidative stress and apoptosis from ischemia and reperfusion injury by targeting ERRFI1. Gene 690, 75-80. doi: 10.1016/j.gene. 2018.12.044

Wen, Z., Mai, Z., Zhu, X., Wu, T., Chen, Y., Geng, D., et al. (2020). Mesenchymal stem cell-derived exosomes ameliorate cardiomyocyte apoptosis in hypoxic conditions through microRNA144 by targeting the PTEN/AKT pathway. Stem Cell Res. Ther. 11:36. doi: 10.1186/s13287-020-1563-8

Wojciechowska, A., Braniewska, A., and Kozar-Kamińska, K. (2017). MicroRNA in cardiovascular biology and disease. Adv. Clin. Exp. Med. 26, 865-874. doi: $10.17219 /$ acem/62915

Woodall, B. P., and Gustafsson, ÅB. (2018). Mesenchymal stem cell-mediated autophagy inhibition. Circ. Res. 123, 518-520. doi: 10.1161/CIRCRESAHA.118. 313592

Wu, T., Chen, Y., Du, Y., Tao, J., Li, W., Zhou, Z., et al. (2018). Circulating exosomal miR-92b-5p is a promising diagnostic biomarker of heart failure with reduced ejection fraction patients hospitalized for acute heart failure. J. Thorac. Dis. 10, 6211-6220. doi: 10.21037/jtd.2018.10.52

Wu, X. D., Zeng, Z. Y., Gong, D. P., Wen, J. L., and Huang, F. (2019). Potential involvement of S1PR1/STAT3 signaling pathway in cardiac valve damage due to rheumatic heart disease. Biotech. Histochem. 94, 398-403. doi: 10.1080/ 10520295.2019.1574028

Xia, Z. Y., Hu, Y., Xie, P. L., Tang, S. Y., Luo, X. H., Liao, E. Y., et al. (2015). Runx2/miR-3960/miR-2861 positive feedback loop is responsible for osteogenic transdifferentiation of vascular smooth muscle cells. Biomed. Res. Int. 2015:624037. doi: 10.1155/2015/624037

Xing, X., Li, Z., Yang, X., Li, M., Liu, C., Pang, Y., et al. (2020). Adiposederived mesenchymal stem cells-derived exosome-mediated microRNA-342-5p protects endothelial cells against atherosclerosis. Aging 12, 3880-3898. doi: 10.18632/aging. 102857

Xue, S., Liu, D., Zhu, W., Su, Z., Zhang, L., Zhou, C., et al. (2019). Circulating MiR-17-5p, MiR-126-5p and MiR-145-3p are novel biomarkers for diagnosis of acute myocardial infarction. Front. Physiol. 10:123. doi: 10.3389/fphys.2019. 00123

Yi, R., Qin, Y., Macara, I. G., and Cullen, B. R. (2003). Exportin-5 mediates the nuclear export of pre-microRNAs and short hairpin RNAs. Genes Dev. 17, 3011-3016. doi: 10.1101/gad.1158803

Yue, Y., Zhang, Z., Zhang, L., Chen, S., Guo, Y., and Hong, Y. (2018). miR-143 and miR-145 promote hypoxia-induced proliferation and migration of pulmonary arterial smooth muscle cells through regulating ABCA1 expression. Cardiovasc. Pathol. 37, 15-25. doi: 10.1016/j.carpath.2018.08.003

Zeng, Z., Li, Y., Pan, Y., Lan, X., Song, F., Sun, J., et al. (2018). Cancer-derived exosomal miR-25-3p promotes pre-metastatic niche formation by inducing vascular permeability and angiogenesis. Nat. Commun. 9:5395. doi: 10.1038/ s41467-018-07810-w

Zhang, C., Zhang, K., Huang, F., Feng, W., Chen, J., Zhang, H., et al. (2018). Exosomes, the message transporters in vascular calcification. J. Cell Mol. Med. 22, 4024-4033. doi: 10.1111/jcmm.13692

Zhang, S., Chuah, S. J., Lai, R. C., Hui, J. H. P., Lim, S. K., and Toh, W. S. (2018). MSC exosomes mediate cartilage repair by enhancing proliferation, attenuating apoptosis and modulating immune reactivity. Biomaterials 156, 16-27. doi: 10.1016/j.biomaterials.2017.11.028

Zhao, J., Li, X., Hu, J., Chen, F., Qiao, S., Sun, X., et al. (2019). Mesenchymal stromal cell-derived exosomes attenuate myocardial ischaemia-reperfusion injury through miR-182-regulated macrophage polarization. Cardiovasc. Res. 115, 1205-1216. doi: $10.1093 / \mathrm{cvr} / \mathrm{cvz} 040$

Zheng, B., Yin, W. N., Suzuki, T., Zhang, X. H., Zhang, Y., Song, L. L., et al. (2017). Exosome-mediated miR-155 transfer from smooth muscle cells to endothelial cells induces endothelial injury and promotes atherosclerosis. Mol. Ther. 25, 1279-1294. doi: 10.1016/j.ymthe.2017.03.031

Zhong, X., Gao, W., Wu, R., Liu, H., and Ge, J. (2019). Dendritic cell exosomeshuttled miRNA146a regulates exosome-induced endothelial cell inflammation by inhibiting IRAK-1: a feedback control mechanism. Mol. Med. Rep. 20, 5315-5323. doi: 10.3892/mmr.2019.10749

Zhu, J., Liu, B., Wang, Z., Wang, D., Ni, H., Zhang, L., et al. (2019). Exosomes from nicotine-stimulated macrophages accelerate atherosclerosis through miR21-3p/PTEN-mediated VSMC migration and proliferation. Theranostics 9, 6901-6919. doi: 10.7150/thno.37357

Zhu, L. P., Tian, T., Wang, J. Y., He, J. N., Chen, T., Pan, M., et al. (2018). Hypoxia-elicited mesenchymal stem cell-derived exosomes facilitates cardiac repair through miR-125b-mediated prevention of cell death in myocardial infarction. Theranostics 8, 6163-6177. doi: 10.7150/thno. 28021

Conflict of Interest: The authors declare that the research was conducted in the absence of any commercial or financial relationships that could be construed as a potential conflict of interest.

Copyright $\odot 2021$ Zheng, Huo, Li, Wang, Piao, Wang, Zhu, Li, Wang and Liu. This is an open-access article distributed under the terms of the Creative Commons Attribution License (CC BY). The use, distribution or reproduction in other forums is permitted, provided the original author(s) and the copyright owner(s) are credited and that the original publication in this journal is cited, in accordance with accepted academic practice. No use, distribution or reproduction is permitted which does not comply with these terms. 